\title{
Monitoring Land Subsidence in a Rural Area Using a Combination of ADInSAR and Polarimetric Coherence Optimization
}

\author{
Zahra Sadeghi, Mohammad Javad Valadan Zoej, and Jan-Peter Muller
}

\begin{abstract}
This paper investigates a combination of advanced differential synthetic aperture radar interferometry (ADInSAR) with different coherence optimization methods. After the launch of satellites with polarimetry capabilities, differential synthetic aperture radar interferometry (DInSAR) is feasible to generate polarimetric DInSAR to enhance pixel phase quality and increase coherent pixel (CP) density. The first method proposed in this paper, modified coherence set-based polarimetry optimization (MCPO), is a modification of a known single-baseline coherence optimization method to optimize coherence of all interferograms simultaneously. The second method, coherence-set based polarimetry optimization (CPO), was presented by Neumann et al., and is an existing revision of the single-baseline coherence optimization technique. The final method, exhaustive search polarimetry optimization, is a search-based approach to find the optimized scattering mechanism introduced by Navarro-Sanchez et al. The case study is the Tehran basin located in the North of Iran, which suffers from a high-rate of land subsidence and is covered by agricultural fields. Usually such an area would significantly decorrelate but applying polarimetric ADInSAR allows us to obtain a more $\mathrm{CP}$ coverage. A set of dual polarization TerraSAR-X images with $9 \times 9$ and $15 \times 15$ as multilook factors were used within the polarimetric ADInSAR procedure. All three coherence optimization methods with two different multilook factors are shown to have increased the density and phase quality of CPs. Moreover, the estimated deformation rates were evaluated using available levelling measurements. MCPO, which is presented in this paper, works more successful than CPO in terms of CPs density, phase quality and deformation accuracy.
\end{abstract}

Index Terms-Advanced differential synthetic aperture radar interferometry (ADInSAR), coherence optimization, polarimetric differential synthetic aperture radar interferometry (DInSAR), polarimetry.

Manuscript received May 17, 2016; revised October 11, 2016 and January 21, 2017; accepted March 16, 2017. This work was supported by the Faculty of Geomatics and Geodesy, K. N. Toosi University of Technology of Iran. All TerraSAR-X images have been provided by DLR in the framework of LAN1335 project. (Corresponding Author: Zahra Sadeghi.)

Z. Sadeghi is with the Faculty of Geomatics and Geodesy, K. N. Toosi University of Technology, Tehran, Iran, and also with the Imaging Group, Mullard Space and Science Laboratory, University College London, Holmbury St Mary, U.K. (e-mail: z.sadeghi@ucl.ac.uk).

M. J. Valadan Zoej is with the Faculty of Geomatics and Geodesy, K. N. Toosi University of Technology, Tehran, Iran (e-mail: valadanzouj@kntu.ac.ir). J.-P. Muller is with the Imaging Group, Mullard Space and Science Laboratory, University College London, Holmbury St Mary, U.K. (e-mail: j.muller@ucl.ac.uk).

Color versions of one or more of the figures in this paper are available online at http://ieeexplore.ieee.org.

Digital Object Identifier 10.1109/JSTARS.2017.2689823

\section{INTRODUCTION}

A DVANCED differential synthetic aperture radar interferometry (ADInSAR) methods estimate the deformation rate of the Earth's land surface accurately using a permanent scatterer selection process [1]. Due to decorrelation, only those pixels that show permanent behavior over a sequence of interferograms, are identified for differential synthetic aperture radar interferometry (DInSAR) processing [2], [3].

Interferometric coherence is the main criterion for the estimation of pixels' phase stability [4], [5]. Coherent Pixels (CPs) are those with coherence values higher than a threshold. The quality and density of CPs in the scene are key factors in success of ADInSAR methods.

Introduction of Polarimetric Synthetic Aperture Radar Interferometry (POLInSAR) was first investigated in [6] to demonstrate how the analysis of interferometric scattering matrix data can improve the performance of SAR interferometry. One of the key ideas is that it is possible to obtain interferograms from all possible linear combinations of polarization states. One advantage of using this combination is the possible improvement of the coherence level using the polarimetric information [7]. As noise level is indirectly related to coherence, consequently the signal-to-noise ratio is improved and the decorrelation terms are decreased [8]. Therefore, using polarimetric information an optimization problem is introduced and solved to obtain the optimum scattering mechanism which leads to the highest interferometric coherence. This approach is based on the intrinsic assumption that there is no systematic impact on the polarimetric dependence of interferometric phase due to deformations or other processes, such as vegetation growth. However, this assumption can be violated frequently in the some agricultural fields and leads to uncertainty in the estimated deformation [9], [10].

Cloude and Papathanassiou first introduced a polarimetric coherence optimization technique [6]. This method allows different polarization stats at the ends of the baseline. A general technique for coherence optimization with equal polarization states was presented by Colin et al. [7]. Given a multibaseline data set in these methods, coherence can be optimized independently for every baseline. This strategy is called single-baseline coherence optimization that leads to different dominant scattering centers depending on the chosen baseline. A more robust polarimetric optimization approach to find the most 
coherent and dominant scatterer is simultaneous optimization of multibaseline coherence. However, both single-baseline and multibaseline approaches entail coherence improvement due to using polarimetry information. Neumann extended singlebaseline coherence optimization to multibaseline and modified the Colin's method with a multibaseline strategy [8]. Another multibaseline coherence optimization technique was proposed by Navarro Sanchez based on using search spaces to increase density of identified CPs and persistent scatterer pixels [1], [11].

Generally, estimation of land deformation using ADInSAR is suggested over areas covered by vegetation due to decorrelation. To the southwest of the Tehran basin (located in northern Iran) there is an area covered primarily by agriculture fields, which is subject to high-rate land subsidence. Our previous works in this case study presented enhanced algorithms based on ADInSAR to address phase unwrapping error due to decorrelation [2], [3]. A combination of ADInSAR and polarimetry information can improve the identified CP density significantly in vegetated areas. In this paper over the Tehran basin, three different multibaseline coherence optimization approaches in case of two different multilook factors were applied. One of those approaches, modified coherence set-based polarimetry optimization (MCPO), was introduced by this research. Then, optimized interferograms were ingested into an ADInSAR technique to select CPs with coherence values higher than a predetermined threshold. Finally the results were compared in terms of CP density and phase quality and validated through available levelling measurements.

This paper is organized as follows. Section II presents a brief review of concepts of POLInSAR and also the three multibaseline polarimetric optimization approaches. In Section III, the study area and the available dataset is introduced. Section IV describes the implementation of combining coherence optimization methods with ADInSAR and shows output velocity maps. The polarimetric optimization methods are compared in terms of $\mathrm{CP}$ density and then are verified through estimation and analysis of noise phase. Moreover, the results are validated using available levelling measurements. Finally, the main conclusions are discussed in Section V.

\section{COHEREnCE Optimization Using POLINSAR}

One of the key ideas of POLInSAR is application of vector interferometry instead of scalar that was already proposed in [6]. In the case of dual-polarization interferometry, considering there is no data from cross-polar channel, the Pauli form of scattering vector for each pixel can be defined as in [6] as follows:

$$
\underline{k}=\frac{1}{\sqrt{2}}\left[S_{H H}+V V, S_{H H}-V V\right]^{T}
$$

$T$ indicates the matrix transposition operation, and $S_{i j}(i, j=$ $H$ or $V$ ) is the complex scattering coefficient for $j$ transmitted, and $i$ received polarization in the HV polarization basis. Using the outer product formed from the scattering vectors $\underline{k}$ and $\underline{k}$ for images I1 and I2, it can be defined in a $4 \times 4$ matrix $\left[T_{4}\right]^{2}$

$$
\left[T_{4}\right]=\left[\begin{array}{cc}
{\left[T_{11}\right]} & {\left[\Omega_{12}\right]} \\
{\left[\Omega_{12}\right]^{* T}} & {\left[T_{22}\right]}
\end{array}\right]
$$

where $\left[T_{11}\right],\left[T_{22}\right]$, and $\left[\Omega_{12}\right]$ are $2 \times 2$ complex matrices given by

$$
\begin{aligned}
{\left[T_{11}\right] } & =\left\langle k_{1} k_{1}^{* T}\right\rangle \\
{\left[T_{22}\right] } & =\left\langle k_{2} k_{2}^{* T}\right\rangle \\
{\left[\Omega_{12}\right] } & =\left\langle k_{1} k_{2}^{* T}\right\rangle .
\end{aligned}
$$

The generalized vector expression for the coherence $\gamma$ is given by

$$
\gamma=\frac{\left|\left\langle\underline{\omega}_{1}^{* T}\left[\Omega_{12}\right] \underline{\omega}_{2}\right\rangle\right|}{\sqrt{\left\langle\underline{\omega}_{1}^{* T}\left[T_{11}\right] \underline{\omega}_{1}\right\rangle\left\langle\underline{\omega}_{2}^{* T}\left[T_{22}\right] \underline{\omega}_{2}\right\rangle}}
$$

where $\underline{\omega}_{1}$ and $\underline{\omega}_{2}$ are normalized complex vectors that can be interpreted as two scattering mechanisms [6]. The scalar complex value for each pixel can be defined as $\mu=\omega^{* T} \underline{k}$, that is a linear combination of the elements of $k$.

Given a multibaseline dataset, in contrast with the independent single-baseline coherence optimization methods, multibaseline methods simultaneously optimize multibaseline coherence. Thus, the average magnitude of the interferometric coherence, $\overline{|\gamma|}=\frac{1}{n} \sum_{i=1}^{n}\left|\gamma_{n}\right|$, is maximized. This approach generally entails lower coherence magnitudes, but the corresponding scattering mechanisms and their interferometric phases are estimated on basis of all available data set and more accurately. Therefore, they are expected to deliver more robust estimates of the optimized interferometric phases and dominant scattering mechanisms [8]. In this research, three different multibaseline coherence optimization methods were used and enforced equal polarimetric signatures of scatterers along all baselines $\left(\underline{\omega}_{1}=\underline{\omega}_{2}\right)$ called equal scattering mechanism.

\section{A. Modified Coherence Set-Based Polarimetry Optimization (MCPO)}

Colin et al. in 2006 proposed a coherence optimization method, which performs the optimization of the coherence using the same complex unitary vector for both antennas. In the interferometric configuration, both antennas as well as the incidence angles are very close, and it is sensible to calculate a coherence for two equal mechanisms. So in practice matrices $T_{11}$ and $T_{22}$ are both coherence matrices of the target seen under very close incidence angles. It is even more suitable in a single-pass acquisition since signals are not affected by temporal decorrelation effects [7]. Using this assumption, the formulation of $\gamma$ was replaced by the following:

$$
\tilde{\gamma}=\frac{\left|\left\langle\underline{\omega}^{* T}\left[\Omega_{12}\right] \underline{\omega}\right\rangle\right|}{\left|\left\langle\underline{\omega}^{* T}\left[T_{12}\right] \underline{\omega}\right\rangle\right|}
$$

where matrix $T_{12}$ is defined as $T_{12}=\left(T_{11}+T_{22}\right) / 2$. The set of all complex coherence is called the coherence set and is written $\Gamma\left(\Omega_{12}, T_{12}\right)$ [7]. It was proved in [12] that this coherence set 
can be defined as follows:

$$
\Gamma\left(\Omega_{12}, T_{12}\right)=\Omega\left(T_{12}^{-\frac{1}{2}} \Omega_{12} T_{12}^{-\frac{1}{2}}\right)
$$

$\Omega(A)$ is the field of values of matrix $A$ and the optimization of $\tilde{\gamma}$ is equivalent to finding a maximum of the numerical range of matrix $A\left(=T_{12}{ }^{-\frac{1}{2}} \Omega_{12} T_{12}{ }^{-\frac{1}{2}}\right)$.

The main process is finding the radius value of matrix $A$ to optimize the coherence. A numerical approach was used to achieve this goal. Initialization is the first step. $\theta=\theta_{0}=$ $\arg (A(1,1)), \quad \theta_{0} \in[0, \pi][7]$. Then, matrices $A_{\theta}$ and $H_{\theta}$ are calculated as follows:

$$
\begin{aligned}
& A_{\theta}=e^{i \theta} A \\
& H_{\theta}=\frac{A_{\theta}+A_{\theta}^{* T}}{2} .
\end{aligned}
$$

The next step is calculating the maximum eigenvalue and corresponding eigenvector $(x)$ of $H_{\theta}$. A new $\theta$ is extracted from $\arg \left(x^{* T} A x\right)$. An iterative process continues to achieve $\theta$ convergence. The associated optimal vector $\omega$ for the coherence is given by $\omega=T^{-\left(\frac{1}{2}\right)} x$.

An MCPO is a modification of Colin's method for this multibaseline approach. Therefore, instead of optimizing each interferogram individually, the mean value of all simplified coherence according to (5) is optimized and the optimal $\underline{\omega}$ is found for all interferograms. Therefore, the optimization function is changed to

$$
\begin{aligned}
H_{\theta} & =\sum_{k=1}^{m} \sum_{j=1 \neq k}^{m} \frac{A_{\theta k j}+A_{\theta k j}^{* T}}{2} \\
\text { where } A_{\theta k j} & =e^{i \theta_{k j}} T_{k j}{ }^{-\frac{1}{2}} \Omega_{k j} T_{k j}{ }^{-\frac{1}{2}}
\end{aligned}
$$

where $k$ and $j$ are related to master and slave images, respectively, and $m$ is the number of all images.

\section{B. Coherence-Set Based Polarimetry Optimization (CPO)}

Neumann et al., in 2008 extended Colin's coherence optimization method for multibaseline case [8]. This method differs slightly from the modified algorithm presented in Section A in the optimization function and initialization. This iterative optimization method can obtain the optimal scattering mechanism from the eigenvector associated with the largest eigenvalue of the matrix $H_{\theta}$ in

$$
H_{\theta} w=\lambda w
$$

where

$$
\begin{aligned}
H_{\theta} & =\sum_{k=1}^{m} \sum_{j=1 \neq k}^{m} A_{k j} e^{-i \theta_{k j}} \\
A_{k j} & =T^{-\frac{1}{2}} \Omega_{k j} T^{-\frac{1}{2}} \quad \text { where } \\
T & =\frac{1}{m} \sum_{i=1}^{m} T_{k k} .
\end{aligned}
$$

Selecting the initial phase shift $\theta$ can be critical with respect to the number of iterations and robustness. At first, initialization is done, $\theta_{k j}=\arg \left(\operatorname{trace} A_{k j}\right)$. Then, in an iterative process, $H_{\theta}$ and eigenvector $w$ corresponding to the highest eigenvalue are computed. The phase shift is improved in each iteration via $\theta_{k j}=\arg \left(w^{* T} A_{k j} w\right)$ until the termination criterion is met. The optimal scattering mechanism vector $\omega$ is calculated from $\omega=T^{-\frac{1}{2}} w /\left(w^{* T} T^{-\frac{1}{2}} w\right)$.

\section{Exhaustive Search Polarimetry Optimization (ESPO)}

Navarro-Sanchez et al., in 2010 parameterized the scattering mechanism vectors and found the optimum coherence via looking in defined search spaces of each parameter. Parametrization of the projection vector guarantees its unitary nature, $|\underline{\omega}|=1$, and ensures that all possible unambiguous values of $\underline{\omega}$ are taken into account [1]:

$$
\underline{\omega}=\left[\cos \alpha, \sin \alpha e^{j \psi}\right]^{T}, \quad\left\{\begin{array}{l}
0 \leq \alpha \leq \pi / 2 \\
-\pi \leq \psi<\pi
\end{array} .\right.
$$

This equation is specific for dual polarization images. The parameterization is presented in [6]. With this expression, the problem is reduced to finding two real values, i.e., $\alpha$ and $\psi$, for optimizing coherence value [1].

\section{CAse Study AREA AND Data Sets}

The Tehran basin, with an area of $2250 \mathrm{~km}^{2}$, is located in the north of Iran. The southwestern portion of the basin is subject to a high-rate land subsidence [2], [3] and is covered by agricultural fields (see Fig. 1). Therefore, due to temporal decorrelation, the coherence of interferograms and then ADInSAR efficiency decline. In order to improve the ADInSAR procedure through coherence optimization to monitor land subsidence in the Tehran basin, dual polarization TerraSAR-X (TSX) images were acquired. A set of eight dual polarization Stripmap TSX images from July 21 to October 6, 2013 with 11 days interval were obtained to test different combinations of different coherence optimization methods and ADInSAR in the rural case study. Fig. 2 illustrates the spatial and temporal baselines of all slave images with respect to the master one. All images involve $\mathrm{HH}$ and VV channels information. Azimuth and slant-range resolution are 6.6 and $1.17 \mathrm{~m}$, respectively.

As can be seen in Fig. 1, the processing is applied over a $2600 \times 2000$ portion of the Tehran basin containing pixels with the highest rate of deformation and also mostly covered by agriculture fields.

\section{EXPERIMENTAL RESULTS USING ADINSAR AND DISCUSSION}

In this section, the performances of coherence optimization methods are compared in terms of $\mathrm{CP}$ density and their results are verified using estimation and analysis of noise phase. Moreover, the estimated deformation rates are validated through available levelling measurements.

In order to estimate coherence, a multilooking process should be applied based on the Equivalent Number of Looks (ENL) in this paper. A multilook factor is calculated with a predefined ENL value, pixel spacing and resolution in azimuth and 


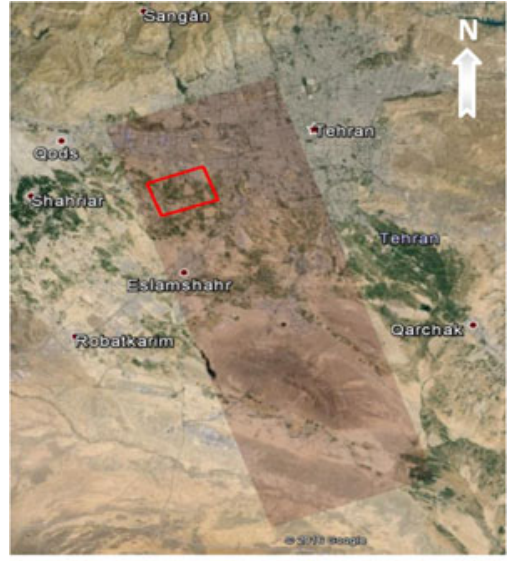

(a)

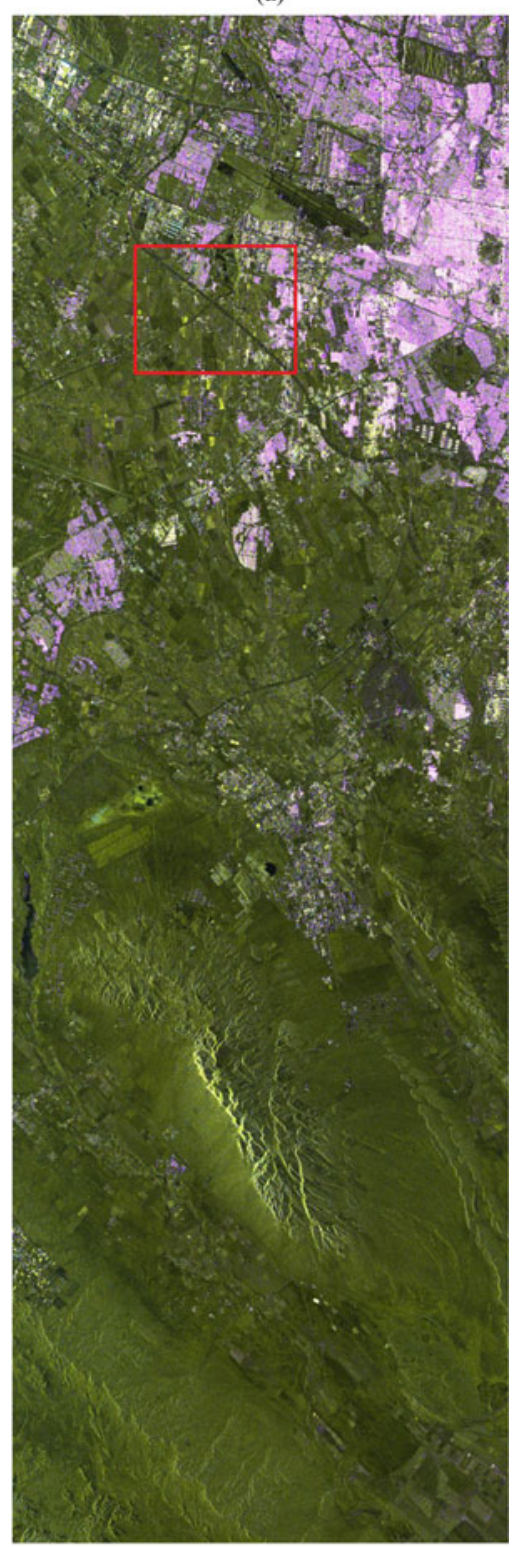

(b)

Fig. 1. (a) Spatial location of master image (shaded rectangle) and case study area (outlined rectangle). (b) Composite RGB of master image (20130903), Channels: $\mathrm{R}=\mathrm{HH}, \mathrm{G}=\mathrm{VV}, \mathrm{B}$ : Absolute value of the difference between channels. The channels are individually scaled based on the mean backscattering value. The black rectangle is the rural case study suffering from land subsidence.

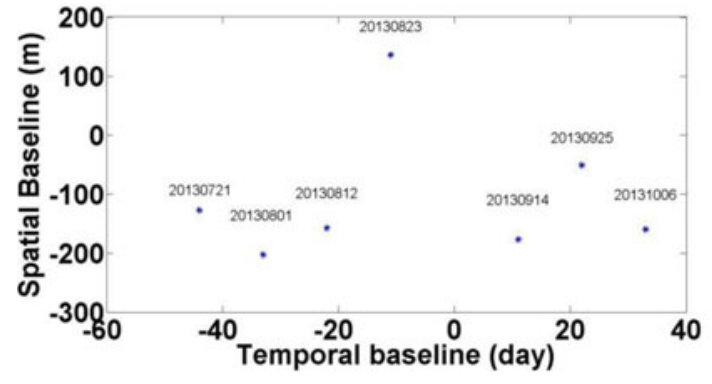

Fig. 2. Temporal and spatial baseline of available dataset.

slant-range orientations. According to the expression derived in [13], with a predefined ENL there is tradeoff between coherence and standard deviation (STD). Windows of $9 \times 9$ and $15 \times 15$ which correspond to ENL of approximately 23 and 64 were chosen to assess effects of a multilook factor in the implemented coherence optimization methods. To keep STDs below 0.09 , coherence thresholds were chosen as 0.6 and 0.4 for $9 \times 9$ and $15 \times 15$ multilook factors, respectively.

Coherence optimization methods and modifying the interferograms to replace the optimum phase were implemented in MATLAB. Interferometric preprocessing and ADInSAR procedures were carried out in Doris and the StaMPS software [14]-[16]. CPs were selected in multilooked interferograms, if at least three interferograms had coherence values above a specified threshold.

Figs. 3 and 4 show deformation velocity maps from the ADInSAR approach for standard single polarimetry information $(\mathrm{HH}$ and VV channel) and optimized coherence via vector polarimetry information. It should be mentioned, different scales for CPs in Figs. 3 and 4 is due to usage of different multilook factors and given an area of approximately $2.2 \mathrm{~m}^{2}$ for each pixel, the area of each multilook CP should be $\frac{15 \times 15}{9 \times 9}$ times larger in Fig. 4 than in Fig. 3. As the figures show, all three coherence optimization methods were successful in increasing the CP density for both the applied multilook factor. Number and percentage of selected CPs in the velocity maps are presented in Tables I and II, respectively.

Numbers of existing pixels for $9 \times 9$ and $15 \times 15$ multilook factors are 64447 and 23316, respectively. The tables show that the VV and ESPO selected the lowest and highest number of CPs in the case study area. A combination of ADInSAR with MCPO, $\mathrm{CPO}$, and ESPO increased the number of identified CPs with respect to the VV channel by approximately $59 \%, 43 \%$, and $72 \%$ for $9 \times 9$ multilooking and $66.3 \%, 59.3 \%$, and $78.6 \%$ for $15 \times 15$ multilooking, respectively. Generally, a $15 \times 15$ multilook factor with a coherence threshold of 0.4 caused a greater coverage of CPs for all three optimization methods comparing against $9 \times 9$, and a combination of the $15 \times 15$ multilook factor with ESPO appears to be the most successful polarimetric ADInSAR method in increasing CP density. Moreover, the MCPO indicates more coverage rather than $\mathrm{CPO}$.

Figs. 5 and 6 illustrate the histograms of $\overline{|\gamma|}$ obtained for single polarimetric channels and the optimized methods. These histograms were calculated using all pixels in the image. It is 


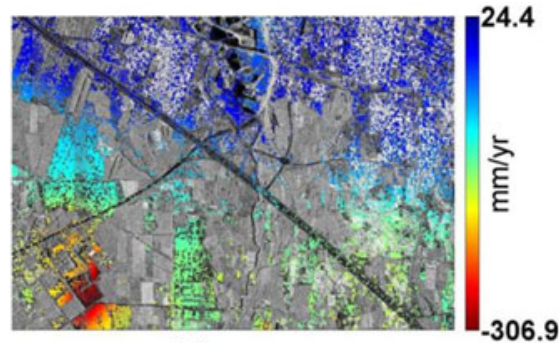

(a)

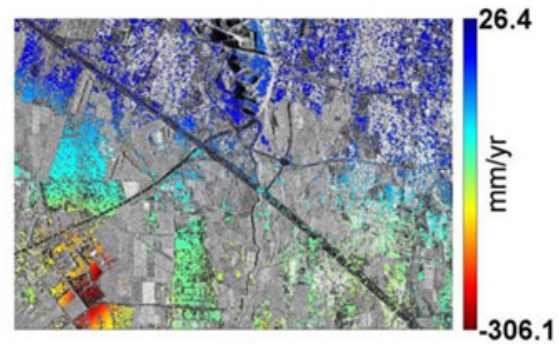

(b)

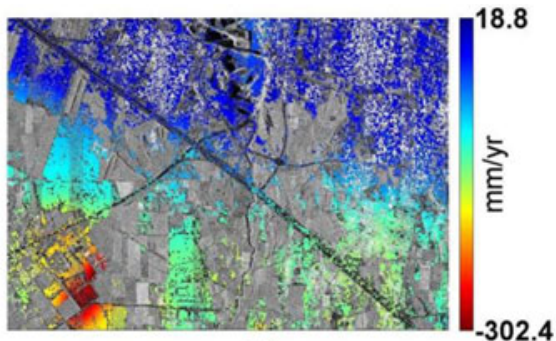

(c)

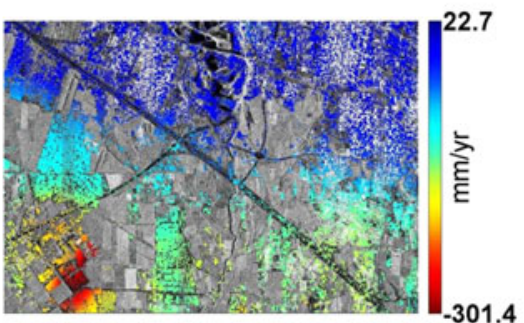

(d)

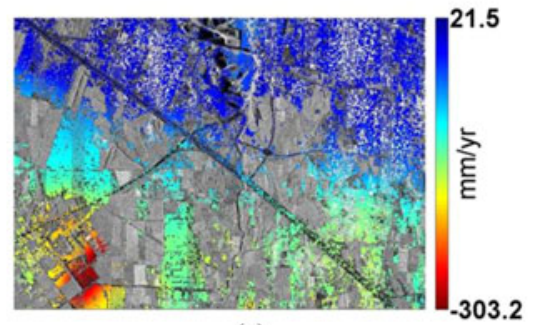

(e)

Fig. 3. Velocity map of selected CP pixels with $9 \times 9$ multilook factor for (a) HH channel, (b) VV channel, (c) optimized channel produced by MCPO, (d) optimized channel produced by CPO, and (e) optimized channel produced by ESPO.

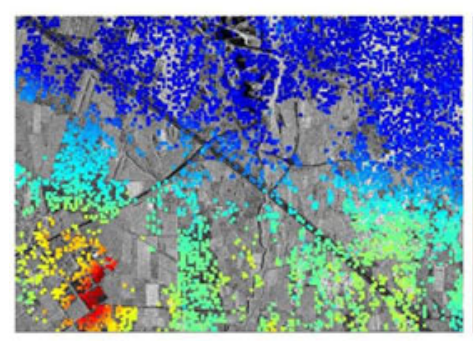

(a)

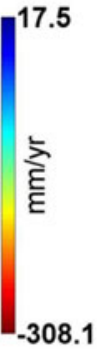

$-308.1$

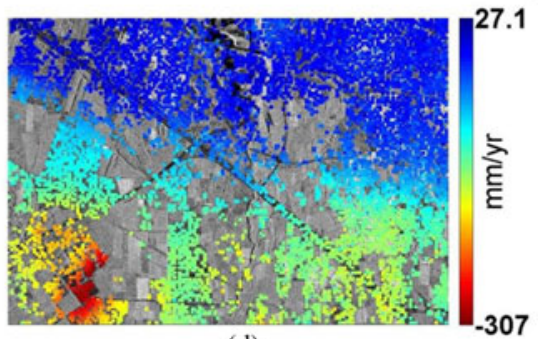

(d)

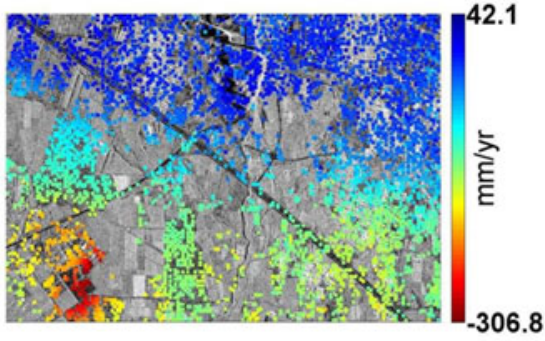

(b)

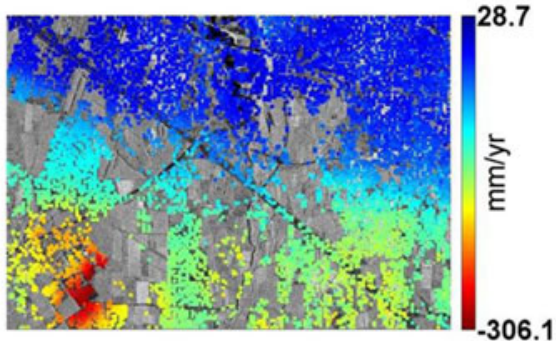

(c)

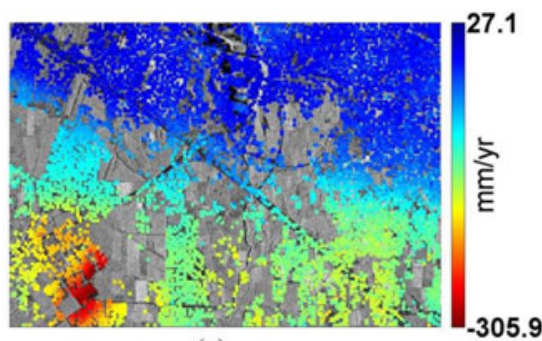

(e)

Fig. 4. Velocity map of selected CP pixels with $15 \times 15$ multilook factor for (a) HH channel, (b) VV channel, (c) optimized channel produced by MCPO, (d) optimized channel produced by CPO, (e) optimized channel produced by ESPO.

TABLE I

Number of SELECTED CPS OF SingLE- AND MULTIPOLARIZATION ADINSAR WiTH TWO DiFFERENT MULTILOOK FACTOR

\begin{tabular}{lccccc}
\hline \hline Multilook Factor & HH & VV & MCPO & CPO & ESPO \\
\hline $9 \times 9$ & 14498 & 12968 & 20570 & 18535 & 22251 \\
$15 \times 15$ & 5922 & 5061 & 8419 & 8062 & 9039 \\
\hline \hline
\end{tabular}

clear that all three optimized methods increased the coherence level with respect to the $\mathrm{HH}$ and VV channel.

In order to assess the phase quality and noise phase level for the polarimetric ADInSAR methods in comparison with the
TABLE II

Percentage of Selected CPs of Single- And Multipolarization ADINSAR WITH Two DIFFERENT MULTILOOK FACTOR

\begin{tabular}{lccccc}
\hline \hline Multilook Factor & HH & VV & MCPO & CPO & ESPO \\
\hline $9 \times 9$ & $22.5 \%$ & $20.1 \%$ & $31.9 \%$ & $28.8 \%$ & $34.5 \%$ \\
$15 \times 15$ & $25.4 \%$ & $21.7 \%$ & $36.1 \%$ & $34.6 \%$ & $38.8 \%$ \\
\hline \hline
\end{tabular}

standard ADInSAR methods, noise phase estimation was applied and analyzed. First, for each ADInSAR method, the CPs were connected to form a network using Delaunay triangulation, and double difference phase measurements were constructed as 


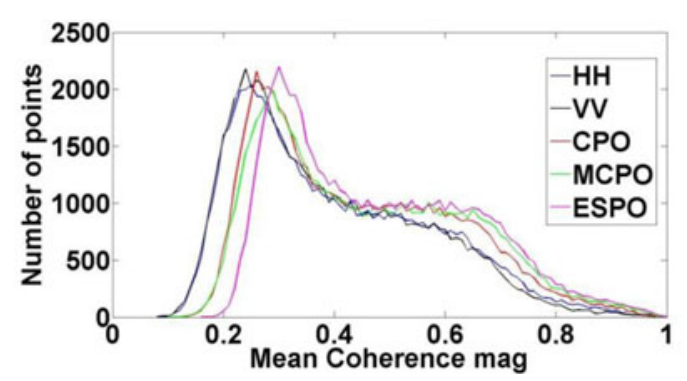

Fig. 5. Histograms of $\overline{|\gamma|}$ for HH, VV, MCPO, CPO, and ESPO for $9 \times 9$ multilook factor.

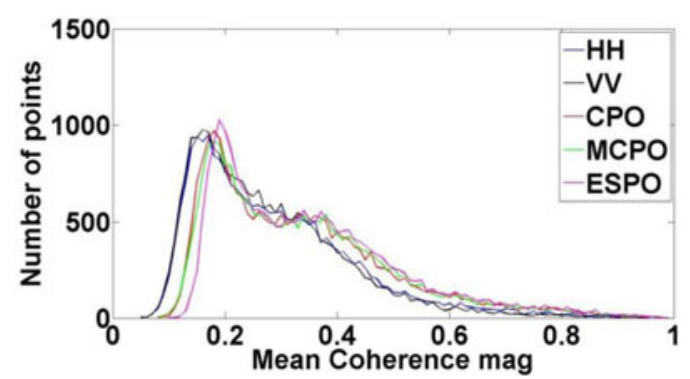

Fig. 6. Histograms of $\overline{|\gamma|}$ for HH, VV, MCPO, CPO, and ESPO for $15 \times 15$ multilook factor.

the differenced phases in space and time for each arc connecting two CPs. For estimating the contribution of noise to the double difference phase, a low-pass filter was used. This filter calculates a weighted mean and then a local fitting of a straight line to estimate smooth phase and then the noise phase after subtraction of the smooth contribution. The weight factors ensure that the nearest points carry the most weight, i.e., the line fit is local and a Gaussian was used for the weighting function. Therefore, for each vector of double difference phase, which contains phase values of an arc in all available interferograms, a vector of contribution of noise was estimated. Subsequently, the maximum magnitude of vector of noise phase, denoted max-noise, and the STD of vector of noise phase, named std-noise, were obtained for each arc.

The estimated max-noise and std-noise for mutual formed arcs between the polarimetric ADInSAR and standard ADInSAR were then compared to verify the impact of polarimetric optimization on the noise phase.

The double difference phase in each interferogram includes the contribution of different phenomena such as deformation, atmospheric error, orbital error and residual DEM error, and noise. Atmospheric error, which results from the atmospheric delay at the two acquisition times, is strongly correlated in space, and the double difference phase measurements between nearby CPs do not contain a significant contribution of atmospheric delay [17], [18]. In order to achieve more reliable results from noise phase estimation, the contribution of orbital and residual DEM error were estimated and subtracted from phase of each CP before triangulation. The phase error from uncertainty in the DEM is proportional to the perpendicular component of the baseline, and was estimated in a least square scene using StaMPS software as this is the only term that would correlate with baseline [15]. The
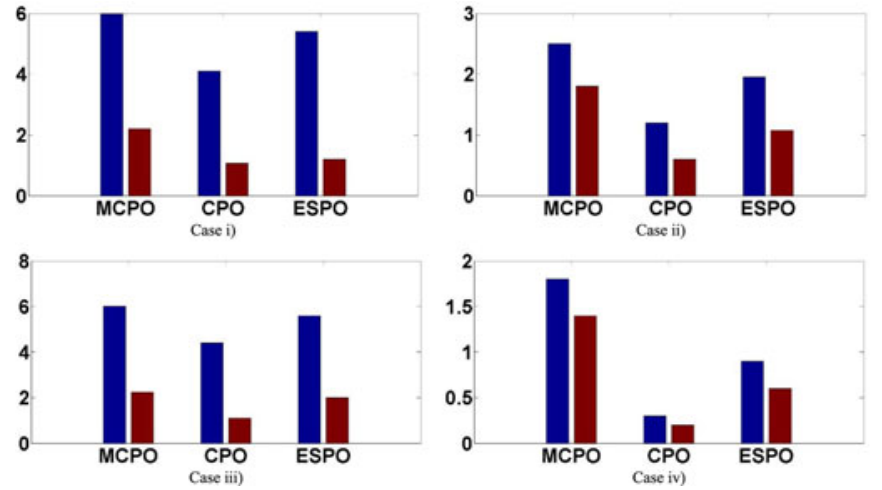

Fig. 7. Percentage of decline in number of arcs with max-noise and stdnoise higher than 0.5 (rad) in three optimum channels with respect to $\mathrm{HH}$ channel for $9 \times 9$ multilook factor in case of (i) residual DEM error reduction, (ii) orbital error reduction, (iii) residual DEM and orbital error reduction and (iv) no reduction from phase of CPs. Blue and red columns show the max-noise and std-noise reduction, respectively.
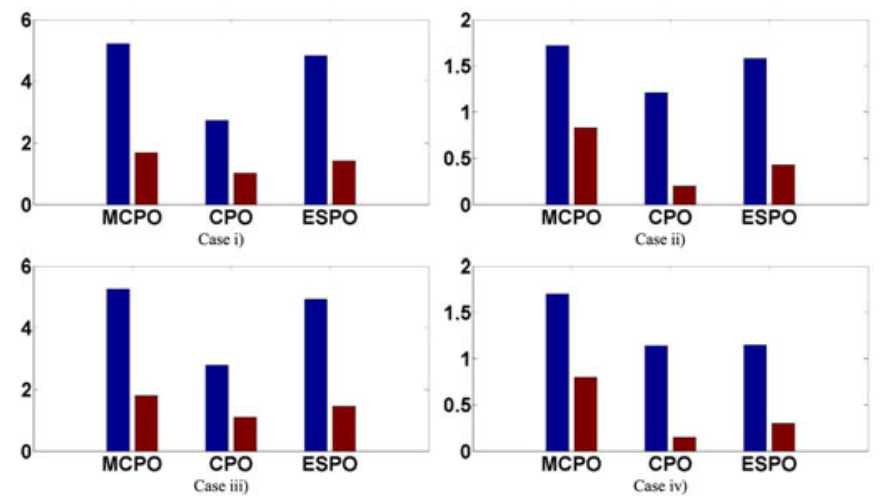

Fig. 8. Percentage of decline in number of arcs with max-noise and stdnoise higher than 0.5 (rad) in three optimum channels with respect to VV channel for $9 \times 9$ multilook factor in case of (i) residual DEM error reduction, (ii) orbital error reduction, (iii) residual DEM and orbital error reduction and (iv) no reduction from phase of CPs. Blue and red columns show the max-noise and std-noise reduction, respectively.

orbital error due to orbit inaccuracies was estimated through fitting a ramp according to StaMPS software [15]. Therefore, obtained max-noise and std-noise for ADInSAR methods were compared in case of 1) residual DEM error reduction, 2) orbital error reduction, 3) residual DEM and orbital error reduction, and 4) no reduction from phase of CPs. Histograms of the estimated noise phase demonstrated that the polarimetric ADInSAR decreases the number of arcs with max-noise and std-noise higher than 0.5 (rad) with respect to standard ADInSAR. Therefore, threshold of 0.5 was selected to compare the noise phase level of the ADInSAR approaches. Figs. 7-10 show the percentages of the declines for two multilook factors of $9 \times 9$ and $15 \times 15$ and the above four different cases.

As can be seen in the above figures, the proposed multibaseline coherence optimization method in this paper, MCPO, is more successful than the other methods, especially CPO, to decrease the noise phase. The second performing method in terms of reducing the noise level is ESPO with achieving slightly less reduction than what it is gained through MCPO method. With 

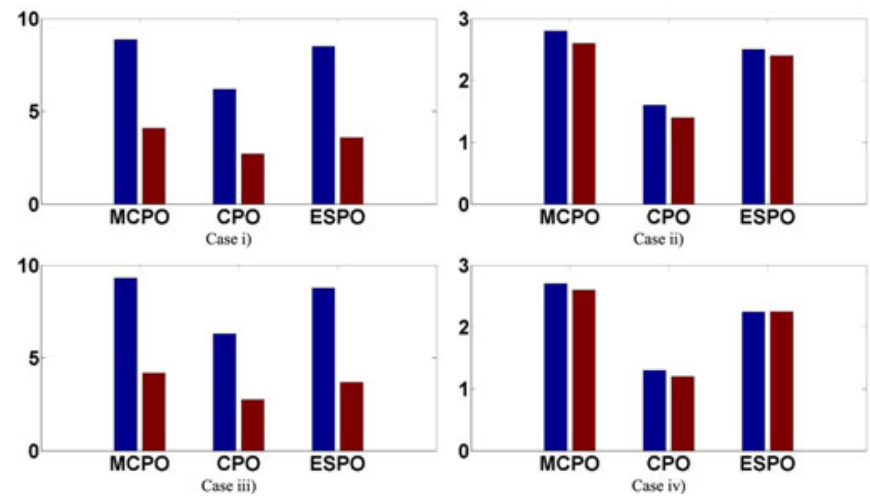

Fig. 9. Percentage of decline in number of arcs with max-noise and std-noise higher than 0.5 (rad) in three optimum channels with respect to $\mathrm{HH}$ channel for $15 \times 15$ multilook factor in case of (i) residual DEM error reduction, (ii) orbital error reduction, (iii) residual DEM and orbital error reduction and (iv) no reduction from phase of CPs. Blue and red columns show the max-noise and std-noise reduction, respectively.
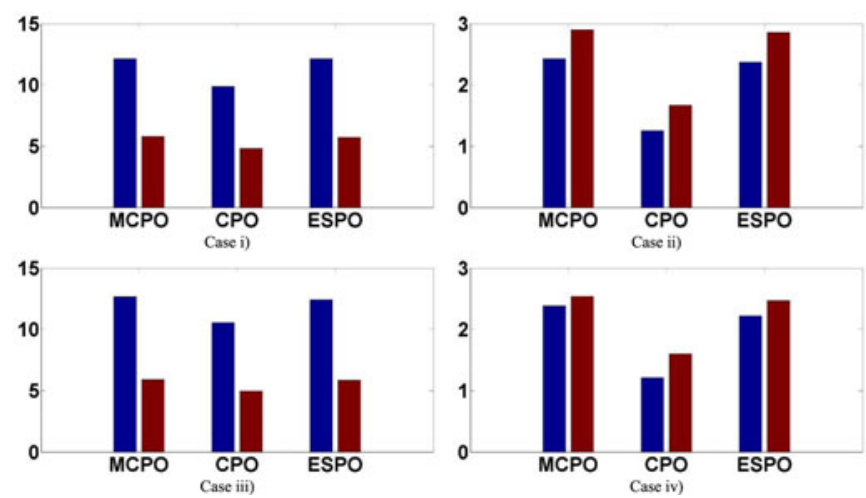

Fig. 10. Percentage of decline in number of arcs with max-noise and std-noise higher than 0.5 (rad) in three optimum channels with respect to VV channel for $15 \times 15$ multilook factor in case of (i) residual DEM error reduction, (ii) orbital error reduction, (iii) residual DEM and orbital error reduction and (iv) no reduction from phase of CPs. Blue and red columns show the max-noise and std-noise reduction, respectively.

regards to the different reduction cases before noise phase estimation, decline of the noise level in case $\mathrm{i}$ is more significant than case iv for the three optimization methods. This can demonstrate the impact of the polarimetric ADInSAR method on the residual DEM error estimation. However, the impact of orbital error removal before noise phase estimation on decreasing the noise level is negligible mainly due to the high spatial correlation of orbital error and its insignificant contribution along the short arcs. Moreover, as it was expected, the results of polarimetric ADInSAR with $15 \times 15$ multilook factor are more successful than $9 \times 9$ multilook factor in reducing the phase noise due to selection of larger factor for multilooking.

In order to quantitatively evaluate the results of ADInSAR, the available levelling observations were used. Because the levelling observations were not collected exactly within the temporal interval of the radar data used in this study (21 July to 6 October 2013), the available observed data were used instead. Levelling measurements were obtained with third-order accuracy during two epochs on 23 September 2013 and 23 April 2014, which is not exactly contemporaneous with the radar

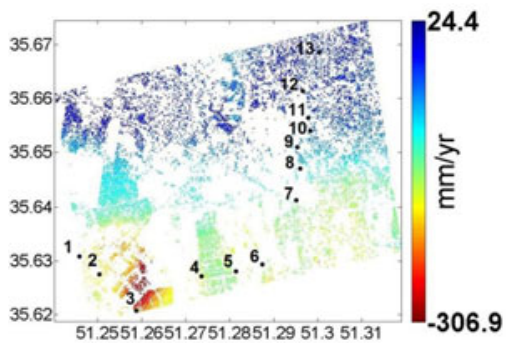

Fig. 11. Spatial location of levelling stations in the case study, the background is deformation map estimated using standard ADInSAR $(\mathrm{HH}$ channel) with $9 \times 9$ multilook factor.
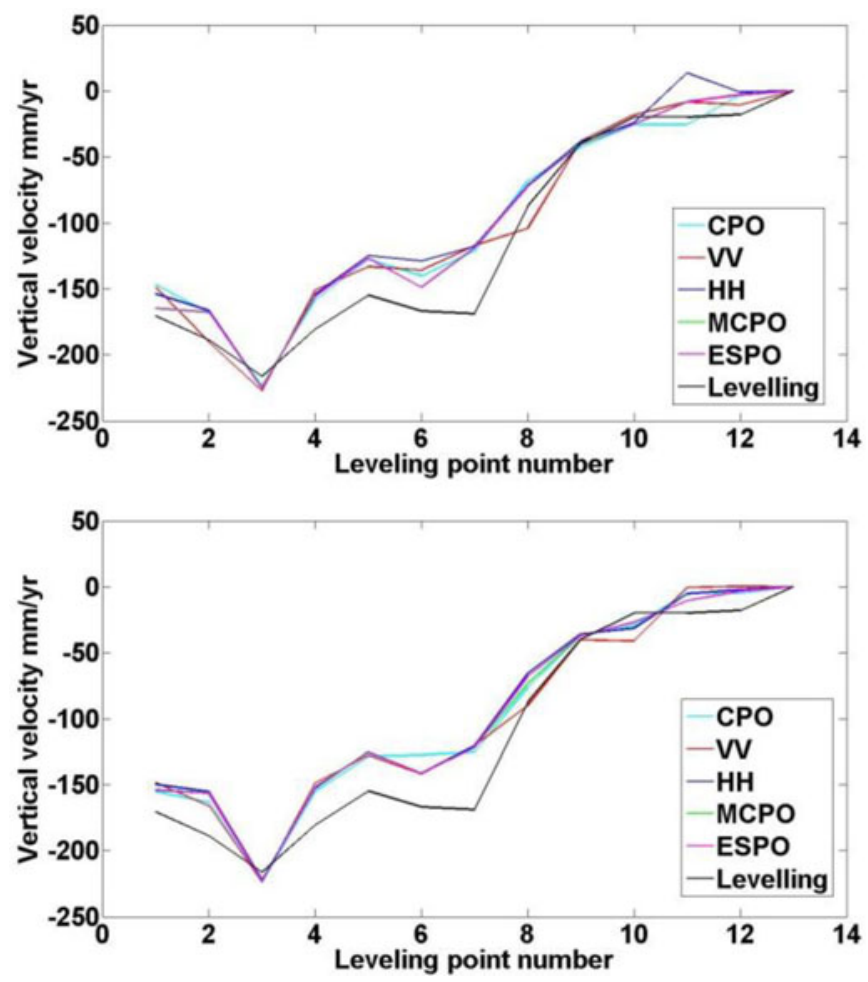

Fig. 12. Comparison of subsidence vertical velocities inferred from the polarimetric and standard ADInSAR and levelling measurements along 13 levelling stations with (a) $9 \times 9$ multilook window (b) $15 \times 15$ multilook window.

data set. There are 13 levelling stations in the case study as can be observed in Fig. 11. Fig. 12 depicts the comparison between the vertical deformation obtained from the polarimetric and standard ADInSAR and the levelling measurements. The reference point is the number 13 levelling station and its nearest $\mathrm{CP}$ for estimated deformation from levelling observations and ADInSAR, respectively. As shown in this figure, the spatial pattern of the subsidence obtained from the ADInSAR methods agree with the levelling measurements along most levelling stations. It should be mentioned that estimated behavior of subsidence for levelling stations by MCPO and ESPO are highly correlated. Table III displays the root mean square error (RMSE) values obtained from all ADInSAR methods and levelling measurements and Tables IV and V show percentages of RMSE improvement for the optimum channels with respect to $\mathrm{HH}$ and VV channel, respectively. As it is obvious, the three coherence optimization methods developed the RMSE values and estimated 
TABLE III

RMSE (MM/YR) VALUES BETWEEN VERTICAL VELOCITIES OF ADINSAR Methods AND LEVELling MEASUREMENTS With Two DifFERENT MULTILOOK FACTORS

\begin{tabular}{lccccc}
\hline \hline Multilook Factor & HH & VV & MCPO & CPO & ESPO \\
\hline $9 \times 9$ & 16.78 & 18.16 & 14.98 & 15.90 & 15.24 \\
$15 \times 15$ & 16.30 & 18.31 & 15.68 & 15.79 & 15.69 \\
\hline \hline
\end{tabular}

TABLE IV

PERCENTAGE OF RMSE IMPROVEMENT With RESPECT TO HH CHANNEL With Two DiFFERENT MuLTILOOK FACTORS

\begin{tabular}{lccc}
\hline \hline Multilook Factor & MCPO & CPO & ESPO \\
\hline $9 \times 9$ & $10.7 \%$ & $5.26 \%$ & $9.16 \%$ \\
$15 \times 15$ & $3.83 \%$ & $3.1 \%$ & $3.74 \%$ \\
\hline \hline
\end{tabular}

TABLE V

PERCENTAGE OF RMSE IMPROVEMENT WITH RESPECT TO VV CHANNEL With Two DifFERENT MultiLOOK FACTORS

\begin{tabular}{lccc}
\hline \hline Multilook Factor & MCPO & CPO & ESPO \\
\hline $9 \times 9$ & $17.5 \%$ & $12.4 \%$ & $16 \%$ \\
$15 \times 15$ & $14.3 \%$ & $13.7 \%$ & $14.27 \%$ \\
\hline \hline
\end{tabular}

the deformation rates more accurately. MCPO, which presented in this paper, was the best algorithm in terms of improving the RMSE values and also more accurate deformation estimation. However, difference between MCPO and ESPO in percentage of RMSE decline is not significant.

It should be mentioned that the different time intervals covered by the radar data (21 July to 6 October 2013) and by the levelling measurements (23 September 2013 to 23 April 2014) can lead to different estimated rates. Therefore, overlapping the time of the levelling observations and the radar data acquisitions leads us to more precisely evaluate the results.

\section{CONCLUSION}

In this paper, high-rate land subsidence in Tehran basin that suffers from temporal decorrelation was studied through a combination of polarimetric information and ADInSAR. Recently, a new generation of radar satellites with different polarimetric channels has created the potential to optimize the interferometric coherence. Thus, using dual polarization TerraSAR-X dataset, three different coherence optimization techniques with $9 \times 9$ and $15 \times 15$ multilook factors were combined with the ADInSAR method.

The resulting velocity map of polarimetric ADInSAR approaches showed all three coherence optimization methods succeeded in increasing the density of selected CPs with respect to single-pol ADInSAR. Results with a $15 \times 15$ multilook factor and coherence threshold of 0.4 illustrated better improvements in CP density when compared with a $9 \times 9$ multilook factor and a coherence threshold of 0.6. Combination of ADInSAR with $\mathrm{MCPO}, \mathrm{CPO}$, and ESPO increased the number of identified CPs with respect to VV channel with approximately 59\%, 43\%, and
$72 \%$ for $9 \times 9$ multilooking and $66.3 \%, 59.3 \%$, and $78.6 \%$ for $15 \times 15$ multilooking, respectively. Therefore, the best coherence optimization method in terms of CP density improvement appears to be ESPO that improved CP coverage significantly with respect to $\mathrm{HH}$ and VV channel.

In order to check the phase quality for polarimetric ADInSAR results with respect to standard ADInSAR, estimation and analysis of noise phase were applied. This analysis for mutual formed arcs between polarimetric ADInSAR and standard ADInSAR showed that the three optimized ADInSAR methods decreased the noise phase level comparing with standard ADInSAR. The best method in terms of phase quality improvement is MCPO, which was presented in this paper. Moreover, the bigger multilook factor which was $15 \times 15$ in this paper entails less noise phase level as it was predicted.

Available levelling measurements in the case study were used to validate the estimated deformation rates through ADInSAR methods. The comparison confirmed that the three polarimetric ADInSAR methods were successful to increase accuracy of deformation estimation with respect to standard ADInSAR. The best method in terms of developing accuracy of deformation estimation is MCPO.

One of the most important findings of this paper was better performance of MCPO in terms of CP density, phase quality, and deformation accuracy in comparison with CPO. Moreover, it was concluded that ESPO was much more successful than $\mathrm{MCPO}$ in increasing $\mathrm{CP}$ density, although superiority of MCPO in terms of phase quality and deformation accuracy over ESPO was not significant.

\section{REFERENCES}

[1] V. D. Navarro-Sanchez, J. M. Lopez-Sanchez, and F. Vicente-Guijalba, "A contribution of polarimetrty to satellite differential SAR interferometry: Increasing the number of pixel candidates," IEEE Geosci. Remote Sens. Lett., vol. 7, no. 2, pp. 276-280, Apr. 2010.

[2] Z. Sadeghi, M. J. Valadan Zoej, and M. Dehghani, "An improved persistent scatterer interferometry for subsidence monitoring in the Tehran basin," IEEE J. Sel. Topics Appl. Earth Observation Remote Sens., vol. 6, no. 3, pp. 1571-1577, Jun. 2013.

[3] Z. Sadeghi, M. J. Valadan Zoej, M. Dehghani and N. B. Chang, "Enhanced algorithm based on persistent scatterer interferometry for the estimation of high-rate land subsidence," J. Appl. Remote Sens., vol. 6, Sep. 2012, Art. no. 063573.

[4] O. Mora et al., "Linear and nonlinear terrain deformation maps from a reduced set of interferometric SAR images," IEEE Trans. Geosci. Remote Sens., vol. 41, no. 10, pp. 2243-2253, Oct. 2003.

[5] R. Lanari, O. Mora, M. Manunta, J. J. Mallorquì, P. Berardino, and E. Sansosti, "A small-baseline approach for investigating deformations on full-resolution differential SAR interferograms," IEEE Trans. Geosci. Remote Sens., vol. 42, no. 7, pp. 1377-1386, Jul. 2004.

[6] S. R. Cloude and P. Papathanassious, "Polarimetric SAR interferometry," IEEE Trans. Geosci. Remote Sens., vol. 36, no. 5, pp. 1551-1565, Sep. 1998.

[7] E. Colin, C. Titin-Schnaider, and W. Tabbara, "An interferometric coherence optimization method in radar polarimetry for high-resolution imagery," IEEE Trans. Geosci. Remote Sens., vol. 44, no. 1, pp. 167-175, Jan. 2006.

[8] M. Neumann, Ĺ. Ferro-Fami,l and A. Reigber, "Multibaseline polarimetric SAR Interferometry coherence optimization," IEEE Geosci. Remote Sens. Lett., vol. 5, no. 1, pp. 93-97, Jan. 2008.

[9] S. Zwieback and I. Hajnsek, "Influence of vegetation growth on the polarimetric zero-baseline DInSAR phase diversity-implications for deformation studies," IEEE Trans. Geosci. Remote Sens., vol. 54, no. 5, pp. 3070-3082, May 2016. 
[10] S. Zwieback, S. Hensley, and I. Hajnsek, "Assessment of soil moisture effects on L-band radar interferometry," Remote Sens. Environ., vol. 164, pp. 77-89, 2015.

[11] V. D. Navarro-Sanchez et al., "Polarimetric approaches for persistent scatterers interferometry," IEEE Trans. Geosci. Remote Sens., vol. 52, no. 3, pp. 1667-1676, Mar. 2014.

[12] M. Tabb, J. Orrey, T. Flynn, and R. Carande, "Phase diversity: Adecomposition for vegetation parameter estimation using polarimetric SAR interferometry," EUSAR, 2002.

[13] R. Touzi, A. Lopes, J. Bruniquel, and W. P. Vachon, "Coherence estimation for SAR imagery," IEEE Trans. Geosci. Remote Sens., vol. 37, no. 1, pp. 135-148, Jan. 1999.

[14] A. Hooper, P. Segall, and H. Zebker, "Persistent scatterer nterferometric synthetic aperture radar for crustal deformation analysis with application to Vacan Alcedo, Galapagos," J. Geophys. Res., vol. 112, 2007a, Art. no. B07407.

[15] A. Hooper, H. Zebker, P. Segall, and B. Kampes, "A new method for measuring deformation on volcanoes and other natural terrains using InSAR persistent scatterers," Geophys. Res. Lett., vol. 31, pp. 611-615, 2004.

[16] A. Hooper, "Persistent scatterer RADAR interferometry for crustal deformation studies and modeling of volcanic deformation," Ph.D. thesis, Stanford Univ., 2006.

[17] S. Williams, Y. Bock, and P. Fang, "Integrated satellite interferometry: Tropospheric noise, GPS estimates and implications for interferometric synthetic aperture radar products," J. Geophys. Res., vol. 103, pp. 27051-27067, 1998.

[18] C. Colesanti, A. Ferretti, C. Prati, and F. Rocca, "Monitoring landslides and tectonic motions with the permanent scatterers technique," Eng. Geol., vol. 68 , pp. 3-14, 2003.

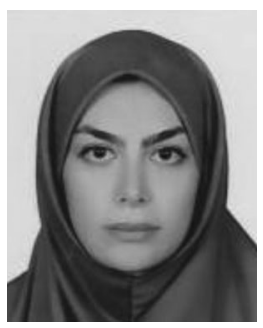

Zahra Sadeghi received the B.S. degree in geomatics engineering from Shahid Rajaee University, Tehran, Iran, in 2008, the M.Sc. degree in remote sensing from K. N. Toosi University of Technology, Tehran, Iran, in 2011. She is currently working toward the $\mathrm{Ph} . \mathrm{D}$. degree in remote sensing at K. N. Toosi University of Technology.

She is visiting Ph.D. student at University College London, London, U.K., working on combination of persistent scatterer InSAR and polarimetric information to improve the identified PS density and then estimate land deformation in rural areas. Since 2009, she has been focusing on applications of different InSAR techniques and presenting enhanced persistent scatterer InSAR methods to monitor land deformation accurately. Her main research interests include satellite remote sensing, InSAR, geohazard monitoring, polarimetric InSAR, SAR tomography, and geodesy.

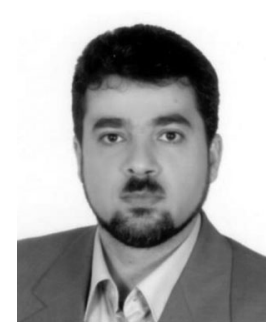

Mohammad Javad Valadan Zoej received the Ph.D. degree in remote sensing and photogrammetry from the University of Glasgow, Glasgow, U.K., in 1997.

$\mathrm{He}$ is currently a Professor with the Faculty of Geodesy and Geomatics, K. N. Toosi University of Technology, Tehran, Iran. His research interests include three-dimensional spatial information extraction from space images, hyperspectral and multispectral image processing, automatic feature extraction, radargrammetry, and interferometry.

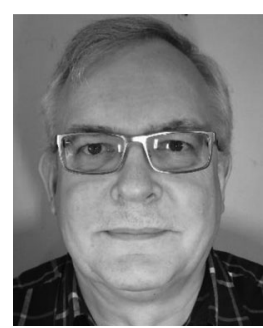

Jan-Peter Muller received the B.S. degree (Hons.) in physics from Sheffield University, Sheffield, U.K. in 1976, the M.Sc. and D.I.C. degrees in atmospheric physics and dynamics from Imperial College London, London, U.K., in 1977, and the Ph.D. degree in planetary meteorology from University College London (UCL), London, U.K., in 1982.

$\mathrm{He}$ is currently the Head of the Imaging Group and a Professor of image understanding and remote sensing with UCL, where he has been a member of the faculty since 1984 . He is currently a Principal Investigator of the EU-FP7 QA4ECV Project, generating an unique 33-year record of Earth's land surface albedo, fAPAR \& LAI from European and U.S. sensors, and the Principal Investigator of the EU-FP7 iMars project to generate a 40-year seamless record of changes on the Martian surface. He has been a member of the NASA Multiangle Imaging Spectroradiometer and Moderate Resolution Imaging Spectroradiometer Science Teams since 1990. He has worked on SAR including interferometric, phase correlation and most recently TOMOSAR since the early 1990s and published extensively in the field. His current research interests include practical applications of machine vision, especially related to Big Data and the role of deep learning applied to remote sensing of the Earth and planets, with a special focus on operational applications of stereo photogrammetry and other three-dimensional imaging technologies for applications in climate modeling, weather forecasting, and planetary exploration. 